\title{
BLOCKCHAIN TECHNOLOGY - THE CONFIDENCE PROTOCOL FOR A NEW REVOLUTION IN KNOWLEDGE SOCIETY
}

\author{
Marian STOICA \\ Bucharest University of Economic Studies, Romania \\ marians@ase.ro \\ Bogdan GHILIC-MICU \\ Bucharest University of Economic Studies, Romania \\ ghilic@ase.ro \\ Marinela MIRCEA \\ Bucharest University of Economic Studies, Romania \\ mmircea@ase.ro
}

\begin{abstract}
The history of humanity has known many turning points in social order, marked by profound changes both in society structure and organization. They were brought on by unprecedented technological progress or by big changes in social thinking and consciousness. Considering their geographical impact, many of them were restricted to country level and only a few produced global changes. The propagation speed for planetary level changes was reduced, leading to slow changes with various consequences in each country. The development of Information and Communication Technology (ICT) has led during the last three decades to profound and extensive changes, marking a passing to a new type of society, based on information, communication and knowledge: the present day knowledge society. Each emergent ICT technology has led to a real revolution. The first four decades of internet have brought upon numerous such revolutions, from daily used electronic mail (e-mail), World Wide Web, social networks and mobile web to newer concepts, technologies and paradigms like big data, data mining, cloud and fog computing, IoT and IoV. All these, corroborated with the financial crisis of early 2000 were nothing but the premises for what is now known as blockchain revolution or internet trust protocol. Since this is a real revolution, in this paper we will show the way blockchain influences (in random order) business/ enterprise architectures, financial services, anti-plagiarism solutions, economy, society, electoral processes and democracy in general. This is why literature mentions "a second era of democracy" or "liquid democracy".
\end{abstract}

Keywords: blockchain, knowledge society, anti-plagiarism, liquid democracy, confidence protocol, e-voting

JEL classification: $\mathrm{O} 3, \mathrm{P} 14$

DOI: $10.12948 / \mathrm{ie} 2019.04 .02$

\section{Introduction}

The transformations inflicted upon the current human society are no different in scope from the ones in the past. What makes them different is the propagation speed over big distances and fast adoption in almost every country. The new globalization economic trend has accelerated the changes, companies seeing the trans-national and trans-frontier idea as an opportunity to develop through new markets, cheaper or better qualified workforce, cheaper and easier accessible raw materials. Business prospects have stimulated companies to invest more in data gathering and transmission infrastructure, which led to a more intense research in the field of 
informatics and telecommunications, which in turn led to an even more increased impact of these technologies upon human society.

Currently, the challenges of knowledge society, global economic crisis, frequent changes on business environment and implicitly on workforce market require an internet trust protocol that meets the new requirements and changes as well as the future development directions of a technological era.

This trust protocol for internet identified in the blockchain technology must be used to build an infrastructure that allows the individual to acquire the following professional competencies (without being limited to them):

- Use modern theories, principles, methods and instruments for analysis of knowledge, objectives, opportunities, benefits, risks, quality, efficiency, innovation in the context of knowledge society;

- Apply modern concepts, principles and paradigms of the knowledge society in various fields in order to ensure sustainability and innovation;

- Use ICT to model, design, implement and manage activities and new organizational paradigms specific to the knowledge society;

- Develop policies that ensure the implementation of newest methods, techniques, paradigms and technologies in knowledge society's economy;

- Scientific research and design of organizational architectures for informational optimization of the context of knowledge society;

- Update and innovate methods and techniques for scientific research in functional infrastructure of virtually organized activities in digital economy.

As argument, we must note that the last two decades are characterized by a series of processes and phenomena that indicate humanity is in a transition phase of its evolution from industrial society to another type of society. Industrial society emerged with the industrial revolution that meant introduction of machines in economy, social division of work and creation of assembly lines. These elements have led to a huge leap forward for society, with implications not only in economy but also in social, cultural and political life. Resources that lie at the base of this society are energy and raw materials. Economic activity is the ensemble of human actions and decisions regarding rational use of resources to create goods and services, according to the needs and economic interests.

Informational and knowledge society is based on another resource, which takes the main stage: information. This doesn't mean that industrial society does not use information or knowledge, but that technological explosion of the last years has turned information into a strategic resource, a new production factor. The speed of knowledge renewal and the exponentially increasing volume have made knowledge an important component of modern economy and a basic organizational principle of the new society. Knowledge has become the most important production factor in modern economy. It constitutes the basis of power exercise, generates increased productivity and ensures business competitiveness. Since and economy can be defined by the type of activity involving most of its worker, we can currently speak of an informational and knowledge economy just as we spoke about industrial economy (when production and processing were dominant activities) or agricultural economy (when most workers were involved in agriculture).

\section{Context of blockchain technology emergence}

The apex of informational technology was not even reached yet and people are already asking: what is next? Studies establish a reference point for informational society the next 20 or 30 years. The question is what new technologies will emerge and follows after this interval. A starting point could be the so called calm technologies and another is the invisible technologies, 
mind power technologies and bio (mechanical, informational, magnetic etc.) or eco technologies [1]. Futurologists have to find credible answers for all these questions. Analyzing the society evolution through the classical data-information-knowledge trident, we can talk about knowledge society and intelligent society. The current state of humanity development may be defined as a new type of society, in a continuous change, developed on the production of informational values, unlike previous societies which were driven by material production. From this we may foresee that the next wave will start around year 2035-2040 and it may be called intelligence and knowledge stage. It will shift the focus on exploiting information to achieve the intelligence level desired by an entity. It will be the time when the capabilities of the human brain will be matched by technology and the concept of bio-techno-system will be generalized - hybrids between biological and technological systems.

This is the current cognitive and technological context, where technology no longer creates prosperity and it doesn't destroy the private life either. Still, in this digital age, technology can be seen in every aspect of human life, at the core of everything good or bad. It is the technology that provides individuals with the possibility to reciprocally value and infringe the other's rights in new and deeper ways [2]. Three years ago we were writing about redefining telework through the cloud computing paradigm [3], highlighting the very important role of trust in the employer-employee relation (Romanian law for regulating telework activities was finally adopted in 2018 - law 18/2018 and it does not mention at all the aspect of trust, teleworking being based only on the accord between the parties). In the context of digital age, security breaches in informational systems and ICT vulnerabilities in current systems (electronic mail, social networks, professional networks and so on) unavoidably affect the trust.

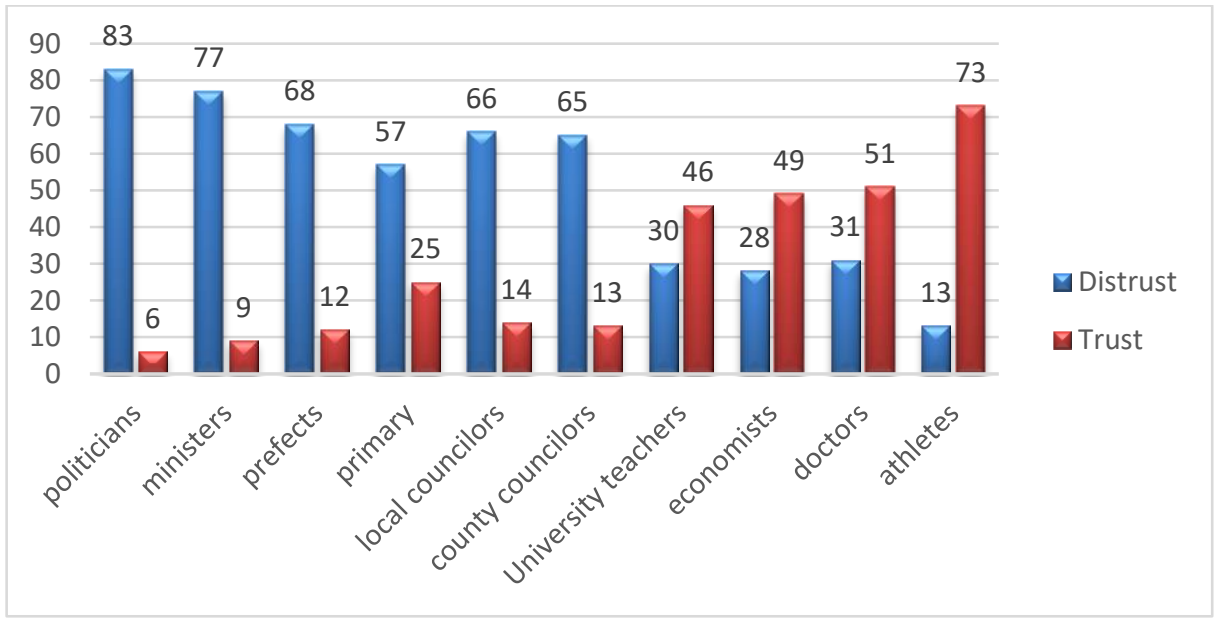

Figure 1. Trust poles for public entities in Romania

From a business perspective, in the digital age, gaining trust means the partner acts according to the four principles of integrity: honesty, consideration, responsibility and transparency. For example, the trust barometer created by the public relation company Edelman in 2018 shows a record decrease of US population trust in public institutions, down to the levels reached during the 2008 recession. Even the ICT industry, once impregnable and with the highest trust level over many years, is now on a downwards trend and seen significant decreases in most countries [4]. The same can be seen in Romania, according to studies published by public polling institutions - for example by Romanian Institute for Evaluation and Strategy (IRES) [5]. On the top of lack of trust sit the public institutions (parliament, government, local government offices, city halls etc.). Highest trust is given to university professors, economists, doctors etc. (figure 1). 
On the other hand, the same source (Edelman) shows that in January 2019 the trust poles have changed drastically in the last year. People have changed the trust regarding the work relations, especially towards their employers. Globally $75 \%$ of people trust that "my employer" does the right thing, significantly higher than for NGOs (57\%), business environment (56\%) and massmedia (47\%) [6].

Al this highlights an acute lack of trust in general and strongly supports the need for trust. In the emergent blockchain world trust derives from the network, from the many blocks that form the chain. In this case trust is related to the integrity and protection of information, not trust in the business endeavors. In other words, anticipating the evolution of production neo-factors from the perspective of modern management, we may argue that those businesses doing their transactions, in part or completely, through blockchain technology will be gain a strategic advantage regarding trust. Trust will take a seat next to information, technology, brand or organizational culture as a vital emerging production factor in a society of knowledge. Blockchain is a protocol that can help internet (re)gain trust.

\section{What is blockchain and how does it work?}

Blockchain technology was invented by a person (or group pf persons) known as Satoshi Nakamoto and published in 2008. The main advantage of this technology is that allows distributing information but not copying it. First field to take advantage of this was cryptocurrency (bitcoin in 2009), but its applicability is more and more desired in other fields. The general understanding is that blockchain is an incorruptible collection of transactions that can be programed not only to record economic exchanges but any virtual values.

In what concerns the inner working of blockchain, the answer might seem complicated, but is can be easier to understand by analogy to a spreadsheet. The sheet may be duplicated millions of times in a network of computers, called nodes, which are programed to update the sheet regularly. In essence, this is the basis of blockchain. As a data structure, blockchain is a linked list, where the links are represented by a summary of block informational content (hash). Thus, each block contains a link to a previous block (a hash of the previous block), a digital stamp with date and time of operations (timestamp) and transaction data (figure 2).

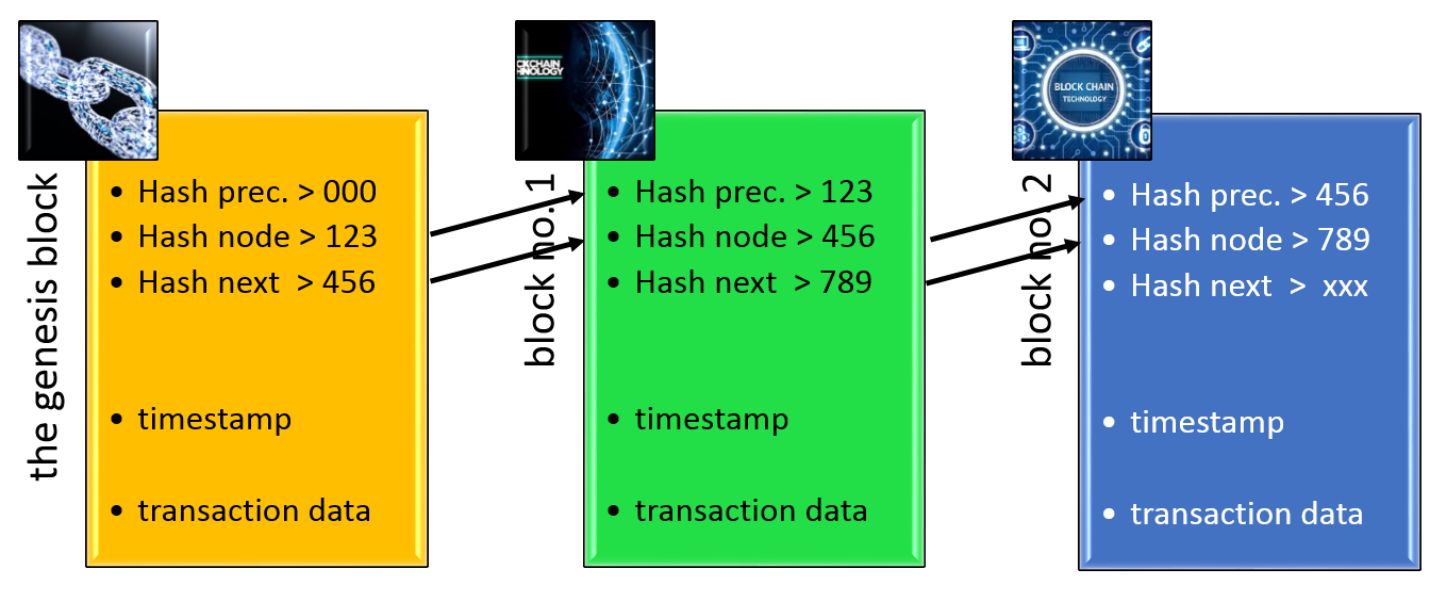

Figure 2. Blockchain mechanism

Another way to see blockchain is as a database distributed in each node. Because the database is stored on many computers, the records are public and easy to verify. There is no centralized version of these records, so they cannot be altered by a hacker. Another advantage comes from the fact that there is no unique entry point, since the database is distributed on many nodes. 
Using blockchain technology, any transaction attribute or information about agents and goods involved is stored in a distributed registry and may be accessed in real-time. The trust in the intermediary is replaced by trust in the basic code and consent rules. These rules define the way a distributed network may reach an agreement, at regular intervals, concerning the status of the shared data it must maintain in order to work in a digital market. Blockchain technology allows a network of economic agents to agree, on regular intervals (10 minutes - the time to create a block) about the real status of shared data. The flexibility of the information represented by these shared data creates a technology with general purpose.

\section{Solutions and possible implications of blockchain}

We must say that blockchain technology is not exclusively owned by the financial industry. There are platforms destined to online writers, with the purpose of guaranteeing the authenticity of texts and fight plagiarism. One such example is the MAVEN project, which is a coalition of experts functioning on an online platform for publishing, publicity and digital distribution, unified under a single media brand [7]. Tens of prizewinning journalists, best writers, sports journalists, top analysts, big causes and foundations bring their organizations in this elite coalition. There are many such antiplagiarism solutions based on blockchain and China is the country that implements them on large scale. These solutions are even embedded in juridical system. According to [8], a court of law recently established in the city of Hangzhou, east China, is set to make use of blockchain technology in the fight against plagiarism for online writers. The use of this technology will save time and reduce the general costs for justice.

Unfortunately, plagiarism exists in every sphere of innovation. In 2018 thousands of blockchain companies emerged and plagiarism scandals became a daily issue. Even success projects are exposed to such accusations, according to [9]. Another performant anti-plagiarism solution is CERTO [10] - an application that uses blockchain technology to verify the existence of any document with a given date in order to prove the authenticity of a product, brand, text and digital literature, video clips or still images, art, illustrations, cartoons, software and so on. CERTO can provide an authenticity certificate by recording the document in the block repository, against plagiarism, fakes and author rights infringements, incorrect use and illegal distribution.

From the current public blockchain platforms dedicated to open source applications worthy mentions are NXT Blockchain Platform (available since 2013) [11] and Ardor Blockchain Platform (available since 2018) - which provides features under the Blockchain-as-a-Service policy [12].

The implications of blockchain are and can go much deeper: from redesigning business processes and enterprise architectures to electronic government, e-voting, digital democracy or liquid democracy. Tapscott speaks of initializing the future (adaptation of the term bootstrapping - the process of initializing an automated sequence or, in this case, of a blockchain) and the seven principles of blockchain economy: network integrity, distributed power, value as incentive, security, confidentiality, rights conservation and inclusion [2].

\section{Conclusions}

Blockchain has become a revolutionary solution for peer-to-peer (P2P) computing a few years ago. Some recognize blockchain as a technology born out of bitcoin, the first major cryptocurrency. Bitcoin was a challenge for the banking industry, but blockchain will change the world in the next 10-15 years more than cloud, robotics or other fast growing technologies. Blockchain analysis has managed to create a new world without intermediaries. Companies can exploit the blockchain technology to build wide computation systems, distributed and open to everybody. Trust is embedded in the blockchain network, each transaction being recorded 
anonymously in the distributed registry or database. The records block changing the payment, music file, medical record or any other file and highlight them in chronological order for ever. No user can secretly modify them because each transaction block must link to the previous block to be valid. This approach has the potential to reorient the entire data and cloud computing industry on short term. Companies and individual consumers may soon store their data in distributed cloud network, based on extremely safe blockchain technology. Cloud computing already brought lower costs, but P2P networks consisting of thousands of personal computers and servers all over the globe bring open cloud computing to an accessible price level for everybody. Thus, we might easily imagine the way computer programs will work on this new infrastructure, which is currently used to validate transactions. We cannot literally accept the computerized analogy in cloud, because block infrastructure cannot completely replace cloud computing, although it divides and democratizes the cloud.

\section{References}

[1] M. Stoica, M. Mircea, B. Ghilic-Micu, C. Uscatu, "From a Smart Education Environment to an Eco-School through Fog \& Cloud Computing in IoT Context," Informatica Economică, vol. 22, no. 4, 2018, pp. 5-14

[2] D. Tapscott, A. Tapscott, "Blockchain Revolution," Ed. Act and Politon, Bucharest, 2017.

[3] B. Ghilic-Micu, M. Stoica, "A Redefinition of Telework through Cloud Computing Telework 2.0," Bulletin of Taras Shevchenko National University of Kyiv. Economics, Ucraine, vol. 46, no. 188, 2016, pp. 16-21

[4] Barometrul de Încredere Edelman 2018, https://www.iqads.ro/articol/41399/barometrulde-incredere-edelman-2018-arata-o-scadere-record-a-increderii, [September 10, 2018]

[5] Barometrul încrederii românilor, IRES 2014-2015, http://www.ires.com.ro, Available: http://www.ires.com.ro/uploads/articole/barometrul-increderii-romanilor.pdf, [May 12, 2018]

[6] 2019 Edelman Trust Barometer, January 20, 2019, Available: https://www.edelman.com/trust-barometer, [March 10, 2019]

[7] MAVEN Cooalition, https://mavencorp.io/press/maven-partners-with-blockchain-basedpo-et-to-empower-publishers-uLPMlewMKkqaKKsqsghxOQ/, [February 9, 2019]

[8] Chinese Internet Court: Blockchain against online plagiarism, December 19, 2018, https://cryptoheroes.ch/chinese-internet-court-blockchain-against-online-plagiarism/

[9] The Loudest Blockchain Plagiarism Scandals, Available: https://www.tokendesk.io/theloudest-blockchain-plagiarism-scandals/ [January 4, 2019]

[10] CERTO Legal, http://www.certo.legal/en/index.html, [February 9, 2019]

[11] Ardor Cryptocurrency and Blockchain Technology, https://ardorplatform.org/, [February 9, 2019]

[12] Nxt - The Blockchain Application Platform, https://nxtplatform.org/, [February 9, 2019] 